\title{
Ab initio calculation of adhesion and potential corrugation of diamond (001) interfaces
}

\author{
Giovanna Zilibotti $^{\mathrm{a}, \mathrm{b}}$, Mauro Ferrario ${ }^{\mathrm{a}, \mathrm{b}, \mathrm{c}}$, Carlo Maria Bertoni ${ }^{\mathrm{a}, \mathrm{b}, \mathrm{c}}$, Maria Clelia Righi ${ }^{\mathrm{a}, \mathrm{b}, *}$ \\ a Dipartimento di Fisica, Università di Modena e Reggio Emilia, Via Campi 213/A, 41100 Modena, Italy \\ ${ }^{\text {b }}$ CNR-Istituto Nanoscienze, Centro $S^{3}$ di Modena, Italy \\ c CNISM, Centro Nazionale Interuniversitario per le Scienze Fisiche della Materia, Italy
}

\section{A R T I C L E I N F O}

\section{Article history:}

Received 30 July 2010

Received in revised form 10 December 2010

Accepted 20 December 2010

Available online 23 December 2010

\section{Keywords:}

Diamond friction

Adhesion

DFT calculations

Dangling bonds

\begin{abstract}
A B S T R A C T
The system consisting of two diamond (001) surfaces in contact was studied by means of planewave/pseudopotential density functional calculations. Different hydrogen coverages, ranging from fully hydrogenation to bare surfaces, were considered. The adhesion energy was calculated as a function of both the separation and the lateral displacement of the two surfaces. The effects of dangling carbon bonds on the adhesion and potential corrugation are quantitatively discussed.
\end{abstract}

(c) 2010 Elsevier B.V. All rights reserved.

\section{Introduction}

The lateral forces experienced by an atomic-size contact when moving along a substrate depend on the corrugation and anisotropy of the potential energy surface (PES) that describes the interaction between the contact and the substrate. The experimental determination of the PES for atomic-size contacts has become possible by using the atomic force microscope (AFM) in a non-contact mode $[1,2]$. The measured PES depends on the shape of the tip and in particular on the composition of its apex, which not always are known. From the theoretical point of view, the PES experienced by an atom or a group of atoms when moving along a surface can be constructed by calculating the slider-substrate interaction potential for different relative positions. In this work, we calculate the PES experienced by an atomic-size contact corresponding to a unit cell of a crystalline diamond surface. Thanks to the periodicity of the system, the results give information on the frictional properties of commensurate interfaces obtained by mating two infinite, flat diamond surfaces. In particular, we analyze the effects of surface passivation on the adhesion and PES corrugation.

Diamond films, artificially grown by chemical vapor deposition (CVD), present excellent frictional properties in humid air and have been increasingly used in many applications both at the macroscopic level and at the nanoscales [3]. However, the reproducibility and the control of the ultralow friction of diamond

\footnotetext{
* Corresponding author at: Dipartimento di Fisica, Università di Modena e Reggio Emilia, Via Campi 213/A, 41100 Modena, Italy.

E-mail address: mcrighi@unimore.it (M.C. Righi).
}

and diamond-like carbon (DLC) have not been fully established yet and intense investigations have been carried out. Previous theoretical studies based on classical molecular dynamics analyzed the effects of temperature [4,5], film composition [6], load [4,7-9], interfacial molecules [10-15] and first principle calculations showed that the presence of adsorbated $\mathrm{H}$ atoms reduces the adhesion of aluminum/diamond [16,17] and diamond/diamond interfaces [18-20]. While diamond films obtained by CVD are mostly hydrogen-terminated, dangling carbon bonds (DBs) can be produced by surface rubbing $[6,21]$. The presence of DBs is considered the cause of the dramatic friction increase observed for diamond in vacuum and in dry conditions, and it has been recently demonstrated by spectroscopic analysis that the ultralow coefficient of friction of diamond in humid environments is due to DBs passivation by water molecules [22].

In this paper we compare the PES calculated for a fully hydrogenated diamond interface with those obtained for hydrogenated interfaces containing dangling carbon bonds (DBs) in different concentrations. Our results show that the presence of DBs strongly increases the adhesion, potential corrugation and anisotropy, demonstrating a direct link between the surface termination and the observed frictional behavior.

\section{Method}

We performed first principles calculations based on density functional theory (DFT) [23]. Ionic species were described by ultrasoft pseudopotentials, and the electronic wavefunctions were expanded in a plane-wave basis. On the basis of test calculations, 
we chose a cut-off energy of 30 Ry for plane-wave expansion. We used the Perdew-Burke-Ernzerhof (PBE) [24] generalized gradient approximation (GGA) to describe the exchange-correlation functional. Tests on bulk diamond revealed that the structural and electronic properties of the material are better described by the PBE approximation than by the local density approximation (LDA) or by other GGA parameterizations [20]. We considered the (001) surface, which is common in CVD grown samples. The bare $\mathrm{C}(001)$ surface presents a $(2 \times 1)$ reconstruction consisting of carbon dimers lying along the [110] direction [25]. This reconstruction is preserved in the hydrogenated $\mathrm{H}-\mathrm{C}(001)$ surface, which, in the most stable phase, presents monohydrogenated dimers [26].

The system of two surfaces in contact, from now on referred to as interface, was simulated by including two slabs 9 layers thick in the same supercell. The slab bottom layers were passivated with $\mathrm{H}$ atoms. We used periodic supercells with a $(2 \times 1)$ in-plane size (the unit cell axes in the [110] and in the [110] directions are $5.06 \AA$ and $2.53 \AA$ long, respectively) and a vertical axis of $29 \AA$ length in the case of surfaces in contact. To simulate two surfaces farther apart than the equilibrium distance, we used a vertical axis of $50 \AA$. We tested the accuracy of the total energy with respect to the number of $\mathbf{k}$ points of the Monkhorst Pack grid [27] adopted to sample the Brillouin zone (BZ), and we found that the results converged with a $(5 \times 10 \times 1)$ grid [20].

The interaction energy $V$ of each couple of surfaces was calculated as: $V=E_{12}^{\text {tot }}-E_{1+2}^{\text {tot }}$, where $E_{12}^{\text {tot }}$ is the total energy of the two surfaces at the equilibrium distance and $E_{1+2}^{\text {tot }}$ is the total energy of two non-interacting surfaces, i.e. separated by $15 \AA$ of vacuum. Negative values of $V$ indicate attractive interaction between the surfaces. The work of adhesion $\gamma$, i.e. the work necessary to separate the two surfaces from contact to infinity [28], can be obtained as $\gamma=-V / A$, where $A$ is the in-plain cell area. The interaction energy was then calculated for different relative lateral positions of the two surfaces, constructing in this way the PES of the sliding interface $V\left(x, y, z_{e q}\right)$. We considered eight grid points per $(2 \times 1)$ cell, representing the relative position of the two surfaces [20]. For each grid point $\left(x_{i}, y_{i}\right)$, a structural relaxation of the system was accomplished by keeping fixed the bottom three layers of the lower slab and only the $(x, y)$ coordinates of the atoms belonging to the three topmost layers of the upper slab. In this way, during the relaxation the distance between the two surfaces (initially set equal to $1.5 \AA$ ) could reach its equilibrium value, $z_{\text {eq }}$.

In order to characterize the interaction between the two surfaces in contact, we calculated the variation of the interaction energy as a function of the separation between the surfaces, $V\left([x, y]_{\min }, z\right)$, where $[x, y]_{\min }$ is the absolute minimum of the lateral PES. For each vertical distance, a relaxation of the system was achieved allowing all the atoms free to move freely, except for those belonging to the three outmost layers of each slab.

\section{Results}

Hydrogen is present in CVD processes, thus diamond surfaces obtained by this growth technique are mostly hydrogen terminated [29]. First principles calculations of surface energy showed, in fact, that the $\mathrm{H}-\mathrm{C}(001)$ surface is more stable than the clean $\mathrm{C}(001)$ one [30]. In the hydrogenated surface the carbon atoms remain arranged in dimer rows as in the clean diamond surface, but the $\pi$-bond between the dimer atoms is replaced by a covalent bond of each surface atom to a hydrogen atom. The calculated Löwdin charges [31] indicate that the hydrogen atom is subject to a slight charge depletion $\delta_{H}=+0.2|e|$, while the carbon atom of the dimer presents an extra charge $\delta_{C}=-0.15|e|$. The presence of a layer of fully saturated polar bonds causes the two fully hydrogenated surfaces to stand far apart: the energy minimization process shows that the two surfaces repulse each other until
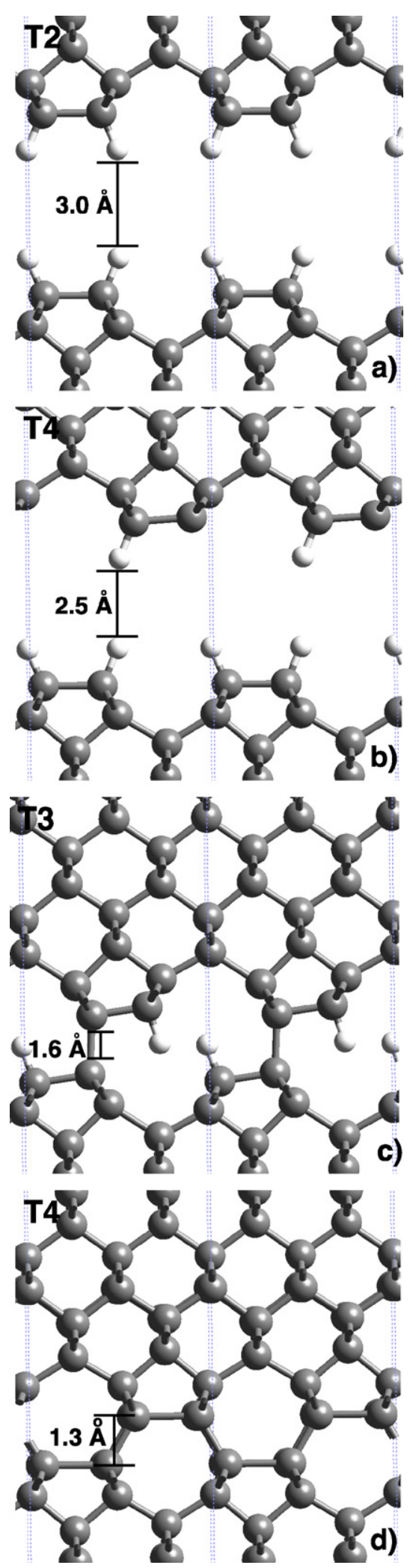

Fig. 1. Lateral view of the relaxed configurations obtained for the hydrogenated interfaces at different coverages: $100 \%$ (a), $75 \%$ (b), $50 \%$ (c), $0 \%$ (d). The snapshots represent the two surfaces at the lateral positions corresponding to the absolute minima of the PESes.

they reach an equilibrium distance of 3.0 A between the hydrogen atoms. The optimized interface configuration is shown in Fig. 1a, while the interaction energy between the two surfaces as a function of their separation is represented in Fig. 2. The $V\left([x, y]_{\min }, z\right)$ 


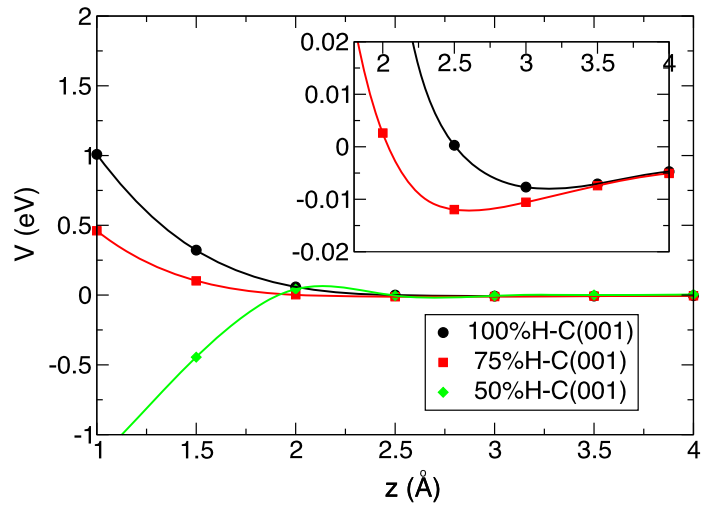

Fig. 2. Interaction energy $V$ as a function of the distance between the surfaces $(z)$. In the inset, the region around the minima is magnified.

Table 1

Adhesion energy for $(2 \times 1)$ cell $\left(V_{\min }\right)$ and equilibrium distance $\left(z_{e q}\right)$ obtained for each considered interface at the grid point corresponding to the minimum of the PES. The value of the maximum corrugation of each PES $\left(\Delta V_{\max }\right)$ is calculated as the difference between the minimum and the maximum values of the adhesion energy.

\begin{tabular}{llll}
\hline & $V_{\min }(\mathrm{eV})$ & $z_{\text {eq }}(\AA)$ & $\Delta V_{\max }(\mathrm{eV})$ \\
\hline $100 \% \mathrm{H}-\mathrm{C}(001)$ & -0.008 & 3.0 & 0.004 \\
$75 \% \mathrm{H}-\mathrm{C}(001)$ & -0.01 & 2.5 & 0.08 \\
$50 \% \mathrm{H}-\mathrm{C}(001)$ & -1.29 & 1.6 & 1.28 \\
$\mathrm{C}(001)$ & -6.0 & 1.3 & 6.7 \\
\hline
\end{tabular}

curve obtained for the fully hydrogenated interface presents the typical features of the physisorption interaction. It is, in fact, characterized by a shallow minimum originating from the combination of a highly repulsive interaction at short range and a weak attraction at long range. The adhesion energy at the equilibrium distance is reported in the first row of Table 1. It is well known that DFT calculations based on LDA, or equally on GGA, where the exchange-correlation energy is a functional of the local electronic density, are not able to accurately describe non-local interactions such as the van der Waals (vdw) interaction. Recent theoretical developments allowed to implement vdw within DFT, for a comprehensive review on the state-of-the-art of describing vdw by DFT see Ref. [32]. An exact description of the interaction energies is beyond the scope of this paper, which is mainly focused on the effects of surface passivation that are highlighted by the different trends observed for different concentrations of DBs, which can be considered reliable. Furthermore, we can observe that the description offered by the present calculations is not too far from reality as revealed by comparison with the experiments: the work of adhesion per unit-area we estimate for the hydrogenated interface of $0.010 \mathrm{~J} / \mathrm{m}^{2}$ is in agreement with the one experimentally measured for a diamond-covered tip on a hydrogenated diamond surface, of $0.0102 \pm 0.004 \mathrm{~J} / \mathrm{m}^{2}[33]$.

Beside the fully hydrogenated interface, we considered hydrogenated interfaces with an increasing number of carbon DBs. In particular, we considered a $75 \% \mathrm{H}$ interface coverage (one DB per interface cell), a $50 \% \mathrm{H}$ interface coverage (two DBs per interface cell), and the interface constituted of two clean $(2 \times 1)-C(001)$ surfaces facing each other.

The optimized configuration of the $75 \% \mathrm{H}-\mathrm{C}(001)$ interface is shown in Fig. 1b. The presence of one DB produces a reduction of the surface equilibrium distance. Covalent $\mathrm{C}-\mathrm{C}$ bonds are not established at the interface, however the presence of an unsaturated carbon bond increases the strength of the interaction between the surfaces: we can see in Fig. 2 that the $V\left([x, y]_{\min }, z\right)$ curve calculated for the $75 \% \mathrm{H}-\mathrm{C}(001)$ interface presents a deeper minimum, which is shifted towards shorter distances with respect to the fully hydrogenated interface. The nature of the surface interaction is completely altered by the presence of more than one DB at the interface. The curve corresponding to the $50 \% \mathrm{H}-\mathrm{C}(001)$ interface presents, in fact, a different character from the other curves, with a drop of the interaction potential $V$ at short distances. In this case, in fact, a covalent $\mathrm{C}-\mathrm{C}$ bond is established across the interface during the translation of one surface along the other one (Fig. 1c) with a consequent increase of the adhesion energy at the lateral position where this event takes place (Table 1 ). The formation of a $\mathrm{C}-\mathrm{C}$ bond at the interface during the relaxation process is favored by the short distance between the slabs selected as starting configuration. Finally, we considered the interaction between two bare diamond surfaces. We can see in Fig. 1d that this situation can result in a cold welding of the surfaces, as also indicated by the adhesion energy reported in Table 1.

The increase of the adhesion energy caused by the presence of carbon DBs at the interface is accompanied by a corresponding increase of the PES corrugation. We can see in Table 1 that the maximum corrugation of the hydrogenated interface increases by about one order of magnitude by including a $25 \%$ density of DBs, while the presence of a $50 \%$ density of DBs produces a PES corrugation three orders of magnitude higher. As in the case of $C(001) / C(001)$, where extremely high potential energy barriers, corresponding to the formation and breaking of diamond bonds, have to be overcome to displace one surface with respect to the other one. This situation produces extremely high friction coefficients and severe wear, as observed experimentally during the sliding of diamond surfaces in vacuum $[34,35]$.

We conclude this section by reporting the DB formation energies. The energy necessary to remove a $\mathrm{H}$ atom from a hydrogenated surface can be obtained as $\varepsilon_{D B}=\left(E_{(N-1) H}^{t o t}+\mu_{H}\right)-E_{N H}^{t o t}$, where $E_{N H}^{t o t}$ is the total energy of the adsorbate system containing $N$ hydrogens, and $\mu_{H}$ is the chemical potential of a hydrogen atom. The latter can assume different values that depend on the absolute temperature $T$ and the partial pressure $p$. We choose the zero reference state of $\mu_{H}(T, p)$ to be the total energy of hydrogen in an isolated molecule, i.e. $\mu_{H}(0 K, p)=1 / 2 E_{H_{2}}^{\text {total }}$. In this case, we obtained $\varepsilon_{1}{ }^{s t} D B=2.26 \mathrm{eV}$ for the energy to create the first DB on a fully saturated dimer, and $\varepsilon_{2^{n d} D B}=1.54 \mathrm{eV}$ for the energy to remove the second hydrogen atom from a carbon dimer where a DB is already present, obtaining in this way a clean, $\pi$-bonded dimer. The calculations to obtain $\varepsilon_{D B}$ were performed by considering the spin polarization and the value obtained for $\varepsilon_{1^{s t} D B}$ is in agreement with previous local spin density (LSD) calculations [36,37], if the isolated hydrogen atom is taken as reference. In Table 2 we report the corrections to be added to the above reported values of $\varepsilon_{D B}$ in order to take into consideration the change of the chemical potential with respect to the reference state. The latter is given by [38]:

$$
\begin{aligned}
\Delta \mu_{H}(T, p)= & 1 / 2\left[H\left(T, p^{0}\right)-H\left(0 K, p^{0}\right)-T S\left(T, p^{0}\right)\right. \\
& \left.+k T \ln \left(\frac{p}{p^{0}}\right)\right]
\end{aligned}
$$

where $H$ and $S$ are the enthalpy and the entropy at a given pressure $p^{0}$, respectively. For standard pressure $p^{0}=1 \mathrm{~atm}$ these values are tabulated in thermochemical tables [39]. The energy corrections reported in Table 2 are calculated according to Eq. (1), considering the partial pressure of $\mathrm{H}_{2}$ in air which is typically $5 \times 10^{-7} \mathrm{~atm}$ [40] and different temperatures. It is clear that the energy cost to create a DB is reduced by increasing the temperature or decreasing the partial pressure. 
Table 2

Change of the chemical potential $\Delta \mu_{H}(T, p)$ relative to the reference value at pressure $p=5 \times 10^{-7} \mathrm{~atm}$ for different temperatures.

\begin{tabular}{llll}
\hline$T$ & $\Delta \mu_{H}(T, p)$ & $T$ & $\Delta \mu_{H}(T, p)$ \\
\hline $300 \mathrm{~K}$ & $-0.35 \mathrm{eV}$ & $700 \mathrm{~K}$ & $-0.90 \mathrm{eV}$ \\
$400 \mathrm{~K}$ & $-0.48 \mathrm{eV}$ & $800 \mathrm{~K}$ & $-1.04 \mathrm{eV}$ \\
$500 \mathrm{~K}$ & $-0.62 \mathrm{eV}$ & $900 \mathrm{~K}$ & $-1.19 \mathrm{eV}$ \\
$600 \mathrm{~K}$ & $-0.76 \mathrm{eV}$ & $1000 \mathrm{~K}$ & $-1.34 \mathrm{eV}$ \\
\hline
\end{tabular}

\section{Conclusions}

We investigated the effects of dangling carbon bonds on the adhesion and potential corrugation of (001) diamond interfaces. Our results indicate that diamond surfaces fully terminated by hydrogen present weak adhesion (few $\mathrm{meV} / \mathrm{cell}$ ) and large equilibrium distances where the potential modulations due to the lateral movement of one surface with respect to the other are of small amplitude $(\Delta V=4 \mathrm{meV} /$ cell). The presence of carbon dangling bonds completely alters this picture. Even when an isolated DB per interface cell is present, thus $\mathrm{C}-\mathrm{C}$ bonds are not formed, the potential corrugation increases by one order of magnitude $(\Delta V=80 \mathrm{meV} / \mathrm{cell})$. Thus, the frictional properties of partially hydrogenated diamond surfaces are expected to be significantly worse than that of fully hydrogenated surfaces. Furthermore, when $\mathrm{C}-\mathrm{C}$ bonds are established at the interface, the energy barriers to displace a surface area, as small as a unit cell, become of the order of the $\mathrm{eV}$.

\section{Acknowledgements}

This work is supported by MIUR through PRIN 2007 BL78N3. We thank the DEISA Consortium (www.deisa.eu), funded through the EU FP7 project RI-222919, for support within the DEISA Extreme Computing Initiative.

\section{References}

[1] B.J. Albers, T.C. Schwendemann, M.Z. Baykara, N. Pilet, M. Liebmann, E.I. Altman, U.D. Schwarz, Nature Nanotechnology 4 (2009) 307.

[2] M. Ternes, C.P. Lutz, C.F. Hirjibehedin, F.J. Giessibl, A.J. Heinrich, Science 319 (2008) 1066.
[3] D.S. Grierson, R.W. Carpick, Nano Today 2 (5) (2007) 12.

[4] J.A. Harrison, C.T. White, R.J. Colton, D.W. Brenner, Phys. Rev. B 46 (1992) 9700.

[5] M.J. Brukman, G. Gao, R.J. Nemanich, J.A. Harrison, J. Phys. Chem. C 112 (2008) 9358.

[6] G.T. Gao, P.T. Mikulski, G.M. Chateauneuf, J.A. Harrison, J. Phys. Chem. B 107 (2003) 11082.

[7] J.A. Harrison, R.J. Colton, C.T. White, D.W. Brenner, Wear 168 (1993) 127.

[8] J.A. Harrison, C.T. White, R.J. Colton, D.W. Brenner, Thin Solid Films 260 (1995) 205.

[9] M.D. Perry, J.A. Harrison, J. Phys. Chem. 99 (1995) 9960.

[10] J.A. Harrison, C.T. White, R.J. Colton, D.W. Brenner, J. Phys. Chem. 97 (1993) 25.

[11] J.A. Harrison, D.W. Brenner, J. Am. Chem. Soc. 116 (1994) 10399.

[12] M.D. Perry, J.A. Harrison, Thin Solid Films 290-291 (1996) 211.

[13] M.D. Perry, J.A. Harrison, J. Phys. Chem. B 101 (1997) 1364.

[14] P.T. Mikulski, J.A. Harrison, Trib. Lett. 10 (2001) 29.

[15] G.T. Gao, P.T. Mikulski, J.A. Harrison, J. Am. Chem. Soc. 124 (2002) 7202.

[16] Y. Qi, L.G. Hector Jr., Phys. Rev. B 68 (2003) 201403(R).

[17] Y. Qi, L.G. Hector Jr., Phys. Rev. B 69 (2004) 235401.

[18] Y. Qi, E. Konca, A.T. Alpas, Surf. Sci. 600 (2006) 2955.

[19] S. Dag, S. Ciraci, Phys. Rev. B 70 (2004) 241401.

[20] G. Zilibotti, M.C. Righi, M. Ferrario, Phys. Rev. B. 79 (2009) 075420.

[21] R.J.A. van den Oetelaar, C.F.J. Flipse, Surf. Sci. 384 (1997) L828.

[22] A.R. Konicek, D.S. Grierson, P.U.P.A. Gilbert, W.G. Sawyer, A.V. Sumant, R.W. Carpick, Phys. Rev. Lett. 100 (2008) 235502.

[23] S. Baroni, A. Dal Corso, S. De Gironcoli, P. Giannozzi, http://www.pwscf.org, 2001.

[24] J.P. Perdew, K. Burke, M. Ernzerhof, Phys. Rev. Lett. 77 (1996) 3865.

[25] C. Kress, M. Fiedler, W.G. Schmidt, F. Bechstedt, Phys. Rev. B 50 (1994) 17697.

[26] H. Kawarada, H. Sasaki, A. Sato, Phys. Rev. B 52 (1995) 11351.

[27] Hendrik J. Monkhorst, James D. Pack, Phys. Rev. B 13 (1976) 5188.

[28] Jacob N. Israelachvili, Intermolecular and Surface Forces, Academic, London, 1992.

[29] J. Ristein, Surf. Sci. 600 (2006) 3677.

[30] A.A. Stekolnikov, J. Furthmūller, F. Bechstedt, Phys. Rev. B 65 (2002) 115318.

[31] A. Szabo, N. Ostlund, Modern Quantum Chemistry, Dover, New York, 1996, p. 153.

[32] A. Tkatchenko, L. Romaner, O.T. Hofmann, E. Zojer, C. Ambrosch-Draxl, M. Scheffler, MRS Bull. 35 (2010) 435.

[33] A.V. Sumant, D.S. Grierson, J.E. Gerbi, J.A. Carlisle, O. Auciello, R.W. Carpick, Phys. Rev. B 76 (2007).

[34] M.N. Gardos, S.A. Gabelich, Trib. Lett. 6 (1999) 103.

[35] R.J.A. van den Oetelaar, C.F.J. Flipse, Surf. Sci. 384 (1997) L828.

[36] S. Hong, M.Y. Chou, Phys. Rev. B 55 (1997) 9975.

[37] J. Furthmūller, J. Hafner, G. Kresse, Phys. Rev. B 53 (1996) 7334.

[38] K. Reuter, M. Scheffler, Phys. Rev. B 65 (2001) 035406.

[39] http://webbook.nist.gov/chemistry/form-ser.html, NIST Chemistry WebBook; http://www.codata.org/resources/databases/key1.html.

[40] D.R. Lide, CRC Handbook of Chemistry and Physics, 73rd ed., CRC Press, Boca Raton, Florida, 1992. 